\title{
The dynamical importance of binary systems in young massive star clusters
}

\author{
Richard de Grijs ${ }^{1}$, Chengyuan $\mathbf{L i}^{1,2}$ and Aaron M. Geller ${ }^{3,4}$ \\ ${ }^{1}$ Kavli Institute for Astronomy \& Astrophysics and Department of Astronomy, Peking \\ University, Beijing, China \\ email: grijs@pku.edu.cn \\ ${ }^{2}$ Purple Mountain Observatory, Chinese Academy of Sciences, Nanjing, China \\ ${ }^{3}$ Center for Interdisciplinary Exploration and Research in Astrophysics (CIERA) and \\ Department of Physics and Astronomy, Northwestern University, Evanston, IL, USA \\ ${ }^{4}$ Department of Astronomy and Astrophysics, University of Chicago, Chicago, IL, USA
}

\begin{abstract}
Characterization of the binary fractions in star clusters is of fundamental importance for many fields in astrophysics. Observations indicate that the majority of stars are found in binary systems, while most stars with masses greater than $0.5 M_{\odot}$ are formed in star clusters. In addition, since binaries are on average more massive than single stars, in resolved star clusters these systems are thought to be good tracers of (dynamical) mass segregation. Over time, dynamical evolution through two-body relaxation will cause the most massive objects to migrate to the cluster center, while the relatively lower-mass objects remain in or migrate to orbits at greater radii. This process will globally dominate a cluster's stellar distribution. However, close encounters involving binary systems may disrupt 'soft' binaries. This process will occur more frequently in a cluster's central, dense region than in its periphery, which may mask the effects of mass segregation. Using high resolution Hubble Space Telescope observations, combined with sophisticated $N$-body simulations, we investigate the radial distributions of the main-sequence binary fractions in massive young Large Magellanic Cloud star clusters. We show that binary disruption may play an important role on very short timescales, depending on the environmental conditions in the cluster cores. This may lead to radial binary fractions that initially decline in the cluster centers, which is contrary to the effects expected from dynamical mass segregation.
\end{abstract}

Keywords. stellar dynamics, methods: statistical, binaries: general, Magellanic Clouds, galaxies: star clusters

\section{Introduction}

Star cluster physics has a fundamental bearing on many important fields in contemporary astrophysics. Applications range from their use as tracers of the most violent episodes shaping galaxies and their evolution on cosmic timescales to their unique role in helping us determine the shape of the stellar initial mass function and validating theories of stellar evolution. Observational, theoretical and numerical studies have made rapid progress to date, yet most studies focus only on the properties of the populations of single stars, while binary systems remain largely overlooked.

This is perhaps understandable from a practical perspective: characterizing binaries in distant star clusters is difficult and including them in numerical simulations carries a significant computational cost, to the extent that even the most advanced $N$-body runs often end in unstable configurations and thus lead to crashed programs. In addition, from a theoretical perspective, it is largely unclear how binary systems affect a cluster's colormagnitude diagram (CMD) once we divert our attention from the fairly well-understood 
main-sequence-main-sequence (MS-MS), MS-red-giant-branch (RGB), and RGB-RGB binaries, i.e., the ubiquitous 'normal,' detached binaries. In the latter context, the formation scenarios of so-called 'blue straggler' systems are now finally reaching a concensuslike state, with most scientists agreeing that these objects have likely originated from either binary mergers or mass transfer between components in binary systems.

At the same time, studies of both the field stellar population in the solar nighborhood and the population properties of star-forming regions and some open clusters imply that the binary fractions at the time of starbirth are non-negligible. Mason et al. (1998) derived a binary fraction of $\sim 70 \%$ in massive field stars, although the exact numbers depend on the stellar spectral type considered (Raghavan et al. 2010). In a clustered context, Sana \& Evans (2011) and Sana et al. (2011, 2012a,b) found a binary fraction of around $50 \%$ in young open clusters, including in the 30 Doradus region, which ties in very well with the $\sim 55 \%$ binary fraction quoted by Kobulnicky et al. (2014) for the Cygnus OB2 association. Kouwenhoven et al. (2005) studied the Scorpius OB2 association in detail and provided similar binary fractions, notably separated as a function of stellar spectral type. Binary systems are thus apparently ubiquitous in young star-forming regions, including in regions of clustered star formation, and hence one can expect them to have noticeable effects on the dynamics of their host stellar systems.

\section{Characterizing binary systems in distant star clusters}

Characterizing binary systems in crowded cluster environments is challenging, particularly in distant star clusters. Nevertheless, careful statistical analysis of high-quality CMDs enables quantitative characterization of binary fractions in clusters at distances out to the Magellanic Clouds, 50-60 kpc (e.g., de Grijs et al. 2014; de Grijs \& Bono 2015), using high-resolution Hubble Space Telescope imaging. Unresolved MS-MS binaries captured in a single resolution element will be more luminous than single stars of the same type as the binary's primary component. In fact, unresolved binary systems will attain brightnesses that vary between those defined by the single-star MS and the offset sequence defined by equal-mass (equal-luminosity) MS-MS binary systems. The latter are defined by a binary mass ratio, $q \equiv m_{2} / m_{1}$, of unity, where $m_{1}$ and $m_{2}$ are the binary's primary and secondary components, respectively, and $m_{1} \geqslant m_{2}$. Equal-mass binaries attain luminosities that are twice as bright as those of the equivalent single stars, which thus translates in a binary sequence in a cluster CMD that is offset by 0.75 mag toward brighter magnitudes. $\dagger$ In other words, the presence of a significant fraction of binary systems will broaden a cluster's MS ridge line. If we can accurately characterize that broadening, we will be able to derive the most likely binary fraction.

In practice, characterization of the MS broadening is best done using Monte Carlo simulations (e.g., Hu et al. 2010, their Fig. 6; Milone et al. 2010, their Fig. 4; and references therein), while also allowing for the unavoidable effects owing to photometric uncertainties and line-of-sight blending. Given that the most important observational effect of the presence of unresolved binary systems in a star cluster's CMD is a spread in the magnitude direction, characterization of the MS broadening is most easily accomplished where the MS is shallowest - and for young clusters, that shallowing of the MS occurs well below the MS turn-off region, so that the effects of rapid stellar rotation do not (yet) dominate.

$\dagger$ Note that while the offset in magnitude is most important, for binaries with $q<1$ a small offset in color is also implied. The extent of the latter depends on both the actual mass ratio (e.g., Elson et al. 1998, their Fig. 3) and the photometric color examined. 
$\mathrm{Hu}$ et al. (2010) were among the first to apply this technique to young massive clusters. For their object of interest, the $\sim 2.8 \times 10^{4} M_{\odot}$ compact, $\sim 15-30$ Myr-old cluster NGC 1818 in the Large Magellanic Cloud (LMC), they derived a best-fitting MS (F-star) binary fraction of $55 \% \pm 10 \%$ for $q \geqslant 0.4$. Depending on the mass-ratio distribution governing these binary systems, the cluster's overall binary fraction could be as high as $100 \%$ ( $\mathrm{Hu}$ et al. 2010). This study thus established that young star clusters may indeed host large numbers of binary systems. These binaries are expected to result in a significant dynamical signature, thus implying that analysis of $N$-body simulations of NGC 1818-like clusters without any implementation of binary systems would lead to unreliable physical insights.

\section{Radial migration and binary dissolution}

While $\mathrm{Hu}$ et al. (2010) obtained a first, firm constraint on a young star cluster's closeto-primordial binary fraction, from a dynamical perspective determination of the distribution and impact of these systems as a function of clustercentric radius would be more valuable. Intuitively, one might expect binary systems to behave as single objects in an $N$-body sense, with a somewhat larger cross section than the equivalent single stars. Realistic initial conditions, e.g., a fractal-like stellar distribution determined by the turbulent structure of the interstellar medium (e.g., Goodwin \& Whitworth 2004; Goodwin et al. 2004a,b), were found to lead to rapid dynamical redistribution of the initial stellar masses - on timescales of $\lesssim$ a few Myr (e.g., Allison et al. 2009, 2010) - and hence to a mass-segregated configuration in the inner cluster regions.

Numerical simulations based on realistic initial conditions (i.e., initial substructure and dynamically cool initial configurations) suggest that dynamical mass segregation, at least of the most massive stars, is likely to happen in a crossing time, which is equivalent to the free-fall time defined by the cluster's gravitational potential. Given that NGC 1818 is 15-30 Myr old (or 5-30 crossing times), one would thus naively expect the cluster to be (dynamically) mass segregated in its core (for confirmation, see de Grijs et al. 2002a,b), with an increasing fraction of binary systems at smaller radii.

However, the F-type stars studied by $\mathrm{Hu}$ et al. (2010), with masses of 1.3-1.6 $M_{\odot}$, are not expected to have already reached a state close to energy equipartition across the cluster, because for these stellar masses the half-mass relaxation time is much longer than the cluster's age. This process of clusterwide dynamical mass segregation is therefore likely still fully underway. However, contrary to dynamical expectations based on initial conditions adopting Plummer spheres, in de Grijs et al. (2013) we first found a hint of an increasing fraction of binary systems in NGC 1818 from the innermost (core) radius out to approximately its half-light radius, which we subsequently confirmed in Li et al. (2013b). In light of our naive initial insights, this was surprising. In fact, a previous, preliminary study of the binary population in NGC 1818 (Elson et al. 1998) had reached the opposite conclusion for binaries with primary masses between $2 M_{\odot}$ and $5.5 M_{\odot}$ and $q \gtrsim 0.7$. From the core to a clustercentric distance equivalent to about 3 core radii, they found a decrease in the binary fraction from about $35 \%$ to $20 \%$. We attributed this discrepant result to blending and the near-vertical morphology of the stellar main sequence within the magnitude range observed by Elson et al. (1998). In Geller et al. (2015; their Fig. 7) we showed that these results are not mutually exclusive in view of the different stellar mass ranges examined by Elson et al. (1998) and Li et al. (2013b).

In $\mathrm{Li}$ et al. (2013b), we used a combination of isochrone fitting and statistical $\chi^{2}$ minimization to re-investigate the binary fraction as a function of radius in NGC 1818, in essence improving the method applied by de Grijs et al. (2013). We also applied the 
same method to NGC 1805, a similarly aged compact LMC cluster. Our results in Li et al. (2013b) exhibit opposite trends as to the binary fractions as a function of radius in the inner regions of both clusters. The binary fraction in NGC 1805 decreases significantly from the cluster's inner core to its periphery. We concluded that while early dynamical mass segregation and the disruption of soft binary systems should be at work in both clusters, time-scale arguments imply that early dynamical mass segregation should be very efficient and, hence, likely dominates the dynamical processes in the core of NGC 1805. Meanwhile, in NGC 1818, the behavior in the core is probably dominated by disruption of 'soft' binary systems, i.e., generally wide binary systems characterized by high kinetic compared to potential energies. We speculate that this may be owing to the higher velocity dispersion in the NGC 1818 core, which creates an environment in which the efficiency of binary disruption is high compared with that of the NGC 1805 core. In other words, by applying the same technique to two young massive clusters in the LMC, we found two opposite radial trends in their binary fractions. This implies that these trends are not driven by the analysis method employed (i.e., our approach is unlikely to introduce a bias one way or the other), but that these trends represent physical reality.

\section{Physical implications}

Our speculation that the puzzling radial trend in NGC 1818 may have been caused by the disription of soft binaries systems was subsequently confirmed on the basis of $N$ body simulations (Geller et al. 2013). Using a grid of sophisticated $N$-body simulations, in Geller et al. (2013) we showed that the observed surface density profile of NGC 1818 (Mackey \& Gilmore 2003) and the radial dependence of the binary frequency (de Grijs et al. 2013; Li et al. 2013b) can be reproduced simultaneously at the cluster's most likely age using $N$-body simulations with both initially smooth or substructured and equilibrium or collapsing stellar populations. The radial distribution of the binary frequency in a rich star cluster can transition smoothly over time from a uniform primordial radial distribution, to one that decreases toward the core at early ages, to one that rises toward the core at later ages. Thus both rising and falling radial distributions in binary frequency can arise naturally from the evolution of a binary population within the same rich star cluster as a consequence of both dynamical disruption and mass segregation of the binaries. Indeed, we managed to reproduce the opposite radial trend observed for NGC 1805 in a subsequent $N$-body study (Geller et al. 2015), drawing from the same initial binary distributions in masses and orbital parameters, although with a different initial cluster mass and half-mass radius.

Our recent analysis of the binary fractions in the young massive LMC clusters NGC 1805 and NGC 1818, as a function of radius, thus confirms the dynamical importance of binary systems in such dense environments (see also Schneider et al. 2014). This thus elevates previously held convictions that binary dynamics are predominantly important in the context of blue straggler formation to a whole new level. We strongly encourage the community, in particular our colleagues with expertise in $N$-body simulations, to take up the gauntlet and henceforth fully embrace the opportunities afforded by inclusion of binary systems in their simulations.

\section{Binaries as diagnostic tool to trace a cluster's dynamical state}

Finally, in Geller et al. (2013), we stumbled upon an interesting diagnostic that could potentially indicate a cluster's dynamical state: see their Fig. 2. Our simulations started from a flat distribution of our NGC 1818-like model binary fraction as a function of 
radius. We found that after one crossing time, the binary fraction decreases toward the cluster core because of the disruption of wide binaries. The higher velocity dispersion in the cluster core compared with its periphery causes binaries in the core to move more rapidly, on average, relative to other stars than their counterparts at larger radii. Therefore, encounters in the core are more energetic and can disrupt tighter binaries, while the higher density results in a higher encounter rate in the core (Leigh \& Sills 2011).

Over time, cluster-wide mass segregation begins to dominate the radial dependence of the binary fraction. As a consequence, the theoretical ' $r_{\text {min }}$ ' values or 'zones of avoidance' (e.g., Mapelli et al. 2004; Ferraro et al. 2012) move radially outward. These radii represent the clustercentric distances inside of which the local dynamical friction timescale (Binney \& Tremaine 1987) for a binary with a mass equivalent to that of the mean binary mass in the cluster is shorter than the simulated time. In other words, $r_{\text {min }}$ predicts the radius inside of which the binaries should experience the effects of dynamical friction and, therefore, migrate toward the cluster center. As a consequence, the binary fraction in the core increases at the expense of that in the cluster's periphery. The observed value of $r_{\mathrm{min}}$ is thus a potential indicator of the degree and extent of mass segregation experienced by a given star cluster.

This behavior of the radial distribution of a cluster's binary fraction is reminiscent of that seen for the radial distribution of blue straggler stars in many Milky Way globular clusters (e.g., Ferraro et al. 2012) as well as in old massive LMC clusters (Li et al. 2013a). Ferraro et al. (2012) indeed used the minima in the radial blue straggler frequency as an indication of the host cluster's dynamical state. They also pointed out that blue straggler formation in a cluster's core may have proceeded differently from that in its outer regions. In view of the higher density in the core and the timescales involved in blue straggler formation, core blue stragglers most likely formed through direct stellar collisions, perhaps mediated by binary encounters, while their peripheral counterparts may well have formed less violently and more slowly through mass transfer between both components of their progenitor binary systems. Once again, this underscores the dynamical importance of binary systems on cluster-wide scales, thus reinforcing our message to take the contributions of binary systems seriously in the context of star cluster dynamics.

\section{Acknowledgements}

RdG and CL acknowledge financial support from the National Natural Science Foundation of China (grants 11073001 and 11373010); RdG also acknowledges travel support from the IAU to attend this conference. CL is supported by Strategic Priority Research Program 'The Emergence of Cosmological Structures' of the Chinese Academy of Sciences (grant XDB09000000). AMG is funded by a National Science Foundation Astronomy and Astrophysics Postdoctoral Fellowship under Award No. AST-1302765. RdG wishes to thank the organizers of IAU Symposium 316 for inviting him to give a review talk, of which this paper is a summary. He specifically thanks Corinne Charbonnel for her competent organizational leadership of the Scientific Organizing Committee.

\section{References}

Allison, R. J., Goodwin, S. P., Parker, R. J., et al. 2009, MNRAS, 395, 1449

Allison, R. J., Goodwin, S. P., Parker, R. J., Portegies Zwart, S. F., \& de Grijs, R. 2010, MNRAS, 407, 1098

Binney, J. \& Tremaine, S. 1987, Galactic Dynamics (Princeton, NJ: Princeton Univ. Press)

de Grijs, R., Johnson, R. A., Gilmore, G. F., \& Frayn, C. M. 2002a, MNRAS, 331, 228 
de Grijs, R., Gilmore, G. F., Johnson, R. A., \& Mackey, A. D. 2002b, MNRAS, 331, 245

de Grijs, R., Li, C., Zheng, Y., et al. 2013, ApJ, 765, 4

de Grijs, R., Wicker, J. E., \& Bono, G. 2014, $A J, 147,122$

de Grijs, R. \& Bono, G. 2015, AJ, 149, 179

Elson, R. A. W., Sigurdsson, S., Davies, M., Hurley, J., \& Gilmore, G. 1998, MNRAS, 300, 857

Ferraro, F. R., Lanzoni, B., Dalessandro, E., et al. 2012, Nature, 492, 393

Geller, A. M., de Grijs, R., Li, C., \& Hurley, J. R. 2013, ApJ, 779, 30

Geller, A. M., de Grijs, R., Li, C., \& Hurley, J. R. 2015, ApJ, 805, 11

Goodwin, S. P. \& Whitworth, A. P. 2004, A\&SA, 413, 929

Goodwin, S. P., Whitworth, A. P., \& Ward-Thompson, D. 2004a, A\&AA, 414, 633

Goodwin, S. P., Whitworth, A. P., \& Ward-Thompson, D. 2004b, A\& A, 423, 169

Hu, Y., Deng, L., de Grijs, R., Liu, Q., \& Goodwin, S. P. 2010, ApJ, 724, 649

Kobulnicky, H. A., Kiminki, D. C., Lundquist, M. J., et al. 2014, ApJS, 213, 34

Kouwenhoven, M. B. N., Brown, A. G. A., Zinnecker, H., Kaper, L., \& Portegies Zwart, S. F. 2005, A\& $A, 430,137$

Leigh, N. \& Sills, A. 2011, MNRAS, 410, 2370

Li, C., de Grijs, R., Deng, L., \& Liu, X. 2013a, ApJL, 770, L7

Li, C., de Grijs, R., \& Deng, L. 2013b, MNRAS, 436, 1497

Mackey, A. D., \& Gilmore, G. F. 2003, MNRAS, 338, 85

Mapelli, M., Sigurdsson, S., Colpi, M., et al. 2004, ApJL, 605, L29

Mason, B. D., Henry, T. J., Hartkopf, W. I., ten Brummelaar, T., \& Soderblom, D. R. 1998, $A J, 116,2975$

Milone, A. P., Piotto, G., King, I. R., et al. 2010, ApJ, 709, 1183

Raghavan, D., McAlister, H. A., Henry, T. J., et al. 2010, ApJS, 190, 1

Sana, H. \& Evans, C. J. 2011, IAU Symp., 272, 474

Sana, H., James, G., \& Gosset, E. 2011, MNRAS, 416, 817

Sana, H., de Mink, S. E., de Koter, A., et al. 2012a, Science, 337, 444

Sana, H., Dunstall, P. R., Hénault-Brunet, V., et al. 2012b, in: Proc. Scient. Mtg in Honor of Anthony F. J. Moffat, ASP Conf. Ser. 465, 284

Schneider, F. R. N., Izzard, R. G., de Mink, S. E., et al. 2014, ApJ, 780, 117 